\title{
Mycoplasma genitalium: whole genome sequence analysis, recombination and population structure
}

\author{
Maria C. Fookes ${ }^{1 *}$, James Hadfield ${ }^{1}$, Simon Harris ${ }^{1}$, Surendra Parmar ${ }^{2}$, Magnus Unemo ${ }^{3}$, Jørgen S. Jensen ${ }^{4}$ \\ and Nicholas R. Thomson ${ }^{1,5^{*}}$
}

\begin{abstract}
Background: Although Mycoplasma genitalium is a common sexually transmitted pathogen causing clinically distinct diseases both in male and females, few genomes have been sequenced up to now, due mainly to its fastidious nature and slow growth. Hence, we lack a robust phylogenetic framework to provide insights into the population structure of the species. Currently our understanding of the nature and diversity of $M$. genitalium relies on molecular tests targeting specific genes or regions of the genome and knowledge is limited by a general undertesting internationally. This is set against a background of drug resistance whereby M. genitalium has developed resistance to mainly all therapeutic antimicrobials.

Results: We sequenced 28 genomes of Mycoplasma genitalium from temporally (1980-2010) and geographically (Europe, Japan, Australia) diverse sources. All the strain showed essentially the same genomic content without any accessory regions found. However, we identified extensive recombination across their genomes with a total of 25 regions showing heightened levels of SNP density. These regions include the MgPar loci, associated with host interactions, as well as other genes that could also be involved in this role. Using these data, we generated a robust phylogeny which shows that there are two main clades with differing degrees of genomic variability. SNPs found in region $\mathrm{V}$ of $23 \mathrm{~S}$ rRNA and parC were consistent with azithromycin/erythromycin and fluoroquinolone resistances, respectively, and with their phenotypic MIC data.

Conclusions: The sequence data here generated is essential for designing rational approaches to type and track Mycoplasma genitalium as antibiotic resistance increases. It represents a first approach to its population genetics to better appreciate the role of this organism as a sexually transmitted pathogen.
\end{abstract}

Keywords: Mycoplasma genitalium genomics and phylogenetics, STIs, Azithromycin resistance, Mycoplasma genitalium

\section{Background}

Mycoplasma genitalium is a pathogenic member of the Mycoplasmataceae restricted to humans, primarily colonizing the urogenital tract, and causing sexually transmitted infections in both males and female [1-3]. It is closely related to the important respiratory tract pathogen $M$. pneumoniae which has a larger genome size of $816 \mathrm{kbp}$ [4] compared to the smaller genome of only $580 \mathrm{kbp}$ for the M. genitalium reference strain

\footnotetext{
*Correspondence: mcf@sanger.ac.uk; nrt@sanger.ac.uk

${ }^{1}$ Pathogen Genomics, The Wellcome Trust Sanger Institute, Wellcome Trust Genome Campus, Hinxton, Cambridge, UK

Full list of author information is available at the end of the article
}

G37 ${ }^{\mathrm{T}}$ [5]. However, all of the 470 proposed M. genitalium coding sequences have orthologues in M. pneumoniae [6]. There are many other human-associated Mycoplasma species, including $M$. hominis, Ureaplasma urealyticum and $U$. parvum. However, the clinical importance of these species is disputed, particularly when they are confined to the lower urogenital tract [7].

M. genitalium causes urethritis in males, and urethritis and cervicitis in females [2]. If not detected and appropriately treated, these infections may result in pelvic inflammatory disease (PID), preterm birth, spontaneous miscarriage, and tubal factor infertility [8]. NAATs are the only feasible, sensitive and specific diagnostic 
methods for $M$. genitalium infections, but access to testing for M. genitalium is limited in many settings [9]. Consequently, epidemiological data regarding $M$. genitalium infections are relatively scarce internationally. This is of concern because $M$. genitalium can develop resistance to nearly all current antimicrobials introduced for treatment and a range of novel antimicrobials, therefore molecular resistance testing is crucial $[10,11]$.

The archetypal M. genitalium isolates, strains $G 37^{\mathrm{T}}$ and M30, were identified in 1980 from two male patients with NGU in the UK [12]. The isolates were recovered from SP4 mycoplasma medium after $>50$ day's incubation and were shown to represent a new species [12]. M. genitalium $\mathrm{G} 37^{\mathrm{T}}$ was selected as the type strain and in 1995, was the first Mycoplasma to be whole genome sequenced [5] showing that it had, at the time, the smallest known genome $(0.58 \mathrm{Mb})$ of any free-living organism, predicted to encode fewer than 500 proteins. As a consequence, M. genitalium was shown to have a reduced metabolic capacity, lacking the enzymes for amino- and fatty acid biosynthesis [5]. This reduced genome, like that of other host- or niche-restricted pathogens, is thought to be the result of a genomic streamlining, where genes that encode functions important for its former lifestyle are lost as the microorganism adapts to a specific host and niche within that host [13, 14]. Other characteristics of the $M$. genitalium genome are the general lack of regulatory systems, the low number of transport systems and, most remarkably, the presence of an expanded family of repetitive chromosomal repeats, known as MgPar regions [5]. The 9 MgPar loci within M. genitalium $G 37^{\mathrm{T}}$ accounted for over $4 \%$ of the genome and have been shown to be involved in antigenic variation $[15,16]$. The MgPar loci represent non-functional fragments of specific genes, $m g p B C$, which are functional and located within a single expression site on the chromosome [5], flanked by MgPar4 upstream and MgPar5 downstream. The genes $m g p B C$ contain trinucleotide tandem repeat regions and have been shown to encode a cytoadhesin (P140) and a conserved hypothetical protein (P110), respectively, both of which are involved in cytoadherence [17]. The MgPar regions represent a reservoir of variation that can be rapidly introduced into the $m g p B C$ gene sequence by recombination between the genes in the expression site and the various MgPar loci [18]. MgpB is a major surface antigen of $M$. genitalium and so this is proposed to increase antigenic diversity as a mechanism of immune evasion, which in turn has been linked to persistence in the human reproductive tract $[16,19]$. In addition, due to the inherent variability of the $m g p B C$ genes they have been used as the basis of most of the established typing schemes for M. genitalium [20, 21].
Despite their importance to human health, there are only 5 fully sequenced $M$. genitalium genomes to date, largely because of the fastidious nature and slow growth of the organism. They include the recent draft genomes of four M. genitalium strains isolated from patients in Denmark, Japan, and Australia [22], which were obtained from axenic cultures raised from single colonies and took up to a year to grow from a swab or urine specimens; a procedure normally involving 2 to 15 passages in Vero cells [23, 24]. To increase our understanding of the population framework and diversity of M. genitalium we sequenced a set of 21 geographically and temporally diverse $M$. genitalium strains ( 1 of them with three isolates from the same patient) as well as other 5 strains available from the ATCC. In doing so we present a phylogenetic snapshot of the species by sequencing a collection of isolates originating from three continents between 1980 and 2010.

\section{Results}

Mycoplasma genitalium isolates, sequencing and variation

A total of $28 \mathrm{M}$. genitalium genomes were sequenced. These included 22 isolates obtained by the Vero cell culture method from 20 patients between 1991 and 2010. Three of these isolates originated from a single French patient collected over a period of 79 days (M6151 day 0; M6090 day 41, and M6312 day 79). Two strains were from samples from Japanese patients collected in 2003 and three were from Australian patients collected in 2004 and 2010 (summarised in Table 1). In addition, an early passage $M$. genitalium M30 strain was also included that originated from samples collected in the UK in 1980, and finally five additional ATCC reference strains were also sequenced: R32G, TW10-6G, TW10-5G, TW48-5G [25] and UTMB-10G [26], described as being isolated from extragenital samples.

De novo assemblies were generated from the Illumina read data for each isolate (see methods). Twelve of the 28 genomes assembled into a single contig (without scaffolding), with the most fragmented genome comprising only 6 contigs (Additional file 1: Table S1). All genomes were syntenic with respect to the published reference $\mathrm{G}^{\mathrm{T}}{ }^{\mathrm{T}}$ [5] and ranged in size from 579,938 to $586,920 \mathrm{bp}$, with the most extreme differences seen for isolates M6327, which was 138 bp shorter and M6713, which was $6844 \mathrm{bp}$ longer than the $\mathrm{G} 37^{\mathrm{T}}$ reference genome (see Table 1 and Additional file 1: Table S1). The additional $6.8 \mathrm{~kb}$ of sequence carried by M. genitalium strain M6713 was entirely explained by the expansion of the number of repeats within a single MgPar locus (see below). In total 8 other isolates exceeded the reference's genome length by $1 \mathrm{~kb}$ or more (see Additional file 1: Table S1) this was all 
Table 1 Main characteristics of the Mycoplasma genitalium sample set $(n=28)$

\begin{tabular}{|c|c|c|c|c|c|c|c|c|}
\hline Sample Name & Country of Origin & Year of Isolation & $A Z M^{b}$ & $\mathrm{ERY}^{\mathrm{b}}$ & $\mathrm{CIP}^{\mathrm{b}}$ & $M X F^{b}$ & $\mathrm{DOX}^{\mathrm{b}}$ & $\mathrm{SOL}^{\mathrm{b}}$ \\
\hline M2282 & Denmark & 1991 & 0.004 & 0.063 & 4 & 0.125 & 0.25 & $<=0.001$ \\
\hline M2300 & Denmark & 1991 & 0.008 & 0.125 & 16 & 0.125 & 0.25 & $<=0.001$ \\
\hline M2341 & Denmark & 1991 & 0.008 & 0.25 & 2 & 0.25 & 1 & $<=0.001$ \\
\hline M30 & UK & 1980 & 0.008 & 0.125 & 8 & 0.125 & 0.5 & $<=0.001$ \\
\hline M6090 ${ }^{\mathrm{a}}$ & France & 1994 & 0.004 & 0.063 & 2 & 0.063 & 0.125 & $<=0.001$ \\
\hline $\mathrm{M} 6151^{\mathrm{a}}$ & France & 1994 & 0.008 & 0.125 & 4 & 0.125 & 0.25 & $<=0.001$ \\
\hline M6257 & Sweden & 2004 & $>8$ & $>16$ & 1 & 0.25 & 0.5 & 2 \\
\hline M6270 & Australia & 2004 & $>8$ & $>16$ & 4 & 0.125 & 0.25 & 0.5 \\
\hline M6280 & Sweden & 1997 & 0.004 & 0.063 & 1 & 0.063 & 0.125 & $<=0.001$ \\
\hline M6283 & Japan & 2003 & 0.004 & 0.063 & 2 & 0.125 & 0.5 & $<=0.001$ \\
\hline M6284 & Japan & 2003 & 0.004 & 0.125 & 4 & 0.125 & 0.25 & $<=0.001$ \\
\hline M6285 & Sweden & 1997 & 0.004 & 0.125 & 1 & 0.125 & 0.5 & $<=0.001$ \\
\hline M6286 & Sweden & 2001 & 0.002 & 0.03 & 2 & 0.03 & 0.25 & $<=0.001$ \\
\hline M6303 & Norway & 2003 & $>8$ & $>16$ & 8 & 0.25 & 2 & 0.5 \\
\hline M6312 & France & 1994 & 0.004 & 0.063 & 8 & 0.125 & 0.25 & $<=0.001$ \\
\hline M6327 & Denmark & 2005 & 0.016 & 0.125 & 8 & 0.25 & 0.25 & 0.002 \\
\hline M6328 & Sweden & 1998 & 0.004 & 0.125 & 2 & 0.125 & 0.25 & $<=0.001$ \\
\hline M6475 & Sweden & 2006 & 0.008 & 0.125 & 1 & 0.5 & 0.5 & $<=0.001$ \\
\hline M6489 & Sweden & 2007 & $>16$ & $>16$ & $>16$ & $>16$ & 1 & 1 \\
\hline M6593 & Norway & 2008 & 16 & $>16$ & 2 & 0.125 & 0.063 & 0.5 \\
\hline M6604 & Denmark & 2009 & 64 & $>16$ & 4 & 0.25 & 0.5 & 2 \\
\hline M6711 & Australia & 2010 & $>16$ & $>=64$ & $>16$ & 8 & 1 & 0.25 \\
\hline M6713 & Australia & 2010 & 0.063 & 0.03 & 0.5 & 0.125 & 0.5 & $<=0.001$ \\
\hline R32G & USA & $1974-1975^{c}$ & ND & ND & ND & ND & ND & ND \\
\hline TW10-6G & USA & $1974-1975^{c}$ & ND & ND & ND & ND & ND & ND \\
\hline TW10-5G & USA & $1974-1975^{c}$ & ND & ND & ND & ND & ND & ND \\
\hline TW48-5G & USA & $1974-1975^{c}$ & ND & ND & ND & ND & ND & ND \\
\hline UTMB-10G & USA & $1986^{d}$ & ND & ND & ND & ND & ND & ND \\
\hline
\end{tabular}

ND: Not determined

${ }^{\text {a }}$ Samples isolated from the same patient

${ }^{\mathrm{b}} \mathrm{MIC}$ values in $\mu \mathrm{g} / \mathrm{ml}$

'Baseman et al. [25]

d Tully et al. [26]

explained by expansion of the number of MgPar regions and/or their repeats or a small number of single base insertions.

Assembled whole genome data showed that there was less than $0.5 \%$ nucleotide divergence, in pairwise comparisons, between any genome sequenced in this study when compared to the $\mathrm{G} 37^{\mathrm{T}}$ reference sequence (range was $0.039 \%$ for strain M6151 to $0.424 \%$ for the M6283; Additional file 1: Table S1). This analysis also showed that all isolates had a similar GC content ( $31.7 \%)$ and the number of predicted coding sequences (CDSs) was 482 for all isolates, identical to the reference $\mathrm{G}^{\mathrm{T}}{ }^{\mathrm{T}}$ genome $[5,27]$.
For completeness we also sequenced the five additional ATCC reference strains: R32G, TW10-6G, TW10-5G, TW48-5G [25] and UTMB-10G [26], described as being isolated from extra-genital samples. The genomes of the five ATCC reference strains showed only minor variation compared to M. genitalium $\mathrm{G} 37^{\mathrm{T}}$, amounting to no whole gene differences and at a maximum of 2-14 SNPs. However, if recombination regions were ignored (using Gubbins; see below for a detailed description of the contribution of recombination to genome variation) then 3 of the 5 strains were in fact identical to $G 37^{\mathrm{T}}$ and 2 samples (UTMP and TW48-5) differed by only a single base pair from the reference strain $G 37^{\mathrm{T}}$. Of note the variation was 
the same $(\mathrm{C}->\mathrm{A})$ and at the same site in both isolates (reference base position 340,583), which represented a synonymous change on the gene MG_279). As these strains are almost identical to the reference strain $\mathrm{G}_{3} 7^{\mathrm{T}}$, our subsequent analyses are based on the remaining 23 genomes sequenced on this work with the addition of the publicly available genomes.

\section{The Mycoplasma genitalium phylogeny}

To construct a whole genome SNP-based phylogeny, sequence reads were mapped to the published genome of the M. genitalium $\mathrm{G} 37^{\mathrm{T}}$ [5] (see methods; Additional file 2: Table S2). The minimum percentage of the $\mathrm{G} 37^{\mathrm{T}}$ genome covered by reads for any of the isolates sequenced here was $98.49 \%$ (isolate M6283). The average depth of mapping coverage for all of the samples was 230× (Additional file 1: Table S1). From the mapped read data, the number of SNPs identified in each sample with respect to the reference ranged from 225 to 2473 for samples M6151 and M6283, respectively, with a total of 11,028 variable sites across the genome. The total number of SNPs per isolate compared to $G 37^{\mathrm{T}}$ is summarised in Additional file 1: Table S1 (including homoplasic SNPs, see below).

M. genitalium, like other Mycoplasma species, has been shown to exhibit high rates of recombination [28]. We used compatibility-based recombination detection methods to assess recombination in the M. genitalium genomes. All methods showed unequivocally strong signals ( $p$-values $<0.01)$ of recombination across all $M$. genitalium genomes sequenced in this study (see methods, data not shown). In order to estimate an accurate phylogeny, we sought to mask out regions of recombination. In total $\sim 60 \%$ of the 11,028 total variable sites identified across all isolates were predicted to be homoplasic by Gubbins (see methods; Fig. 1). Once these SNPs had been masked out, we constructed a maximum likelihood (ML) whole genome phylogeny based on the remaining total number of 4196 variable sites across the 26 isolate genomes (Fig. 1). Comparing phylogenies constructed with and without recombination removed, it is clear that whilst overall topology of the tree remains the same, the branch lengths were shorter in the phylogeny with recombination removed, as would be expected (Additional file 3: Figure S1).

The phylogenetic tree in Fig. 1 shows that the $M$. genitalium isolates form two main clades comprised of 18 (Clade A) and 7 (Clade B) isolates, respectively. The existence of the two clades is well supported (see Additional file 4: Figure S2 for bootstrap values). We used the software package Bayesian Analysis of Population Structure (BAPS) to identify robust Phylogenetic Groups (PGs) within M. genitalium. BAPS identified two PGs, (probability 1, marginal likelihood of the optimal partition $-37,393.6227$ and a reduced likelihood for any change of group ranging from -267.1 and -1093.5), in agreement with the simple population structure designation except for sample M6280, which was reassigned to Clade B. Unlike many other bacterial genomes and considering all SNPs across the phylogeny, there was no obvious correlation between the root to tip phylogenetic distances and the year of isolation (Additional file 5: Figure S3). Moreover, we also found no clustering either to the country of origin or the year of isolation (Fig. 1).

\section{Genes falling within recombination blocks}

Using Gubbins to identify regions of elevated SNP density (a marker of recombination) it was possible to identify 339 recombination blocks representing independent recombination events across the genomes, with distinct borders. These blocks often overlapped to reveal 25 recombination regions composed of contiguous or overlapping recombination blocks, ranging in size from 1035 to 46,265 bp (Fig. 1; Additional file 2: Table S2).

Looking across all isolates only one recombination block, encompassing 7 genes ranging from gyrA to MG_009, was uniquely associated to any particular clade (Clade B; coordinates 6855..11884; region I in Additional file 2: Table S2; Fig. 1). Some of the regions of recombination appeared as 'hotspots' for recombination because there is evidence of recombination having occurred multiple times at that site (Fig. 1). Based on all isolates mapped to the reference, this data show that as much as $66 \%$ of the genome of $\mathrm{G}^{2} 7^{\mathrm{T}}$ falls under a recombination block from one or more of our sequenced set of samples. On average, $50 \%$ of each isolate's genome is predicted to be recombinant. In total, $375 \mathrm{CDSs}$ were encoded within recombination blocks. The most frequent recombination events were centred around the M. genitalium MgPar4 and MgPar5 loci (Fig. 1), although all of the MgPar loci were hotspots, with the recombination blocks generally extending both upstream and downstream of these loci. Recombination between the MgPar loci has been noted previously on a limited number of isolates and shown to include both reciprocal and non-reciprocal intramolecular recombination events $[15,18,29]$. However, it is evident from Fig. 1 that the MgPar regions are not the only regions of recombination across the genome. Other recombination blocks were found to span genes $h m w 2 \quad(257,662 . .278755$; region XIII) and $h m w 1$ and $h m w 3$ (391,075..406322; region XX Additional file 2: Table S2) genes which in $M$. pneumoniae encode proteins involved in cytoadherence $[5,30]$. 


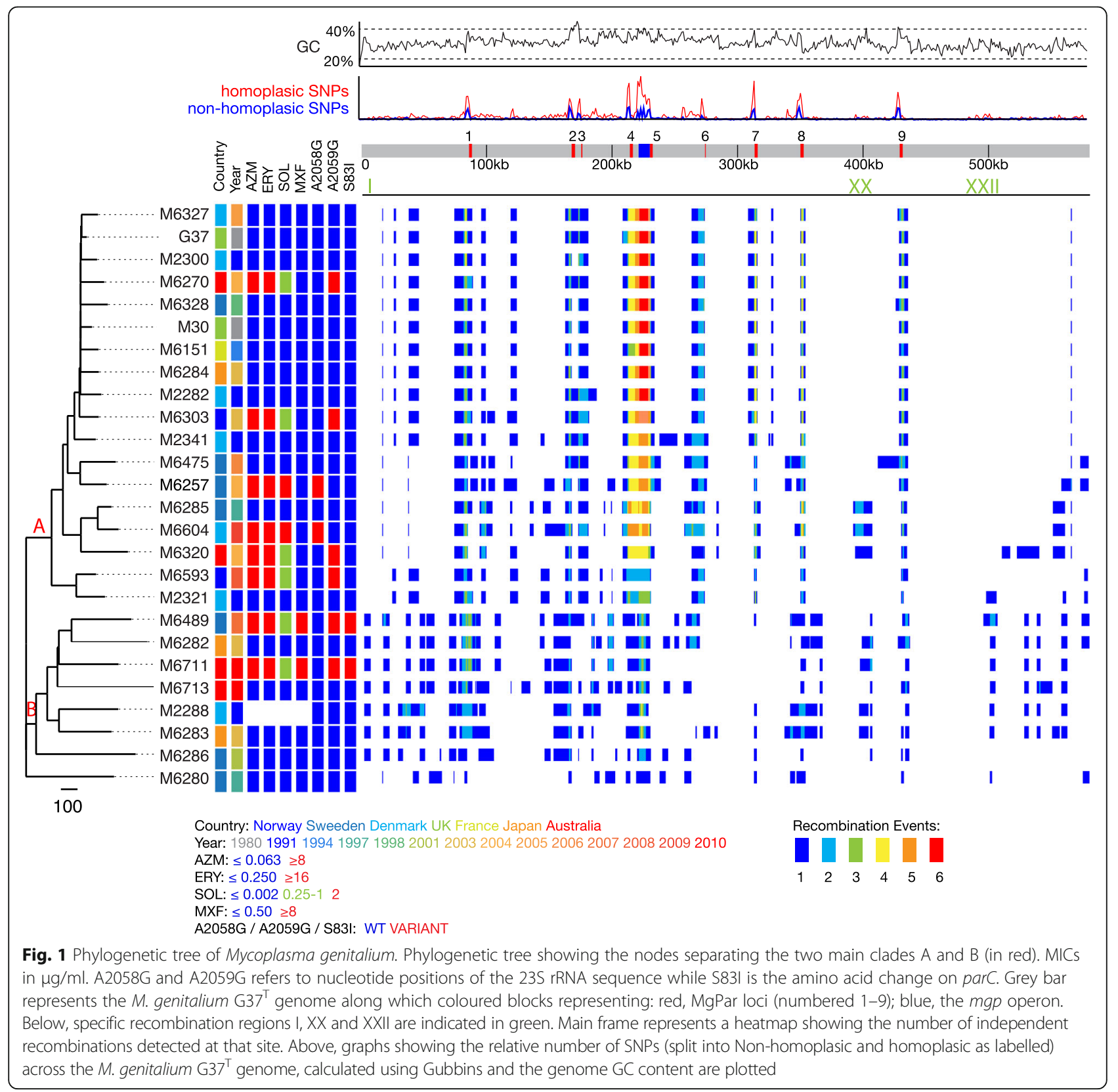

Finally, a region between genes MG_390 and atpD (493,411..504572; region XXII, Additional file 2: Table S2), encompassing MG_395 and MG_397, which is predicted to encode a lipoprotein and a hypothetical protein, respectively, were also found within recombination regions; gene MG_395 shares, 64-74\% nucleotide similarity, to the lipoprotein-encoding genes, MG_067 and MG_068, respectively, which are located immediately upstream of MgPar1 and are included in the recombination block of this first MgPar region. None of these listed loci contained recognised short sequence repeats similar to the ones found within the $m g p B C$ genes.
Recombination between MgPar loci and the mgpABC expression site

The MgPar regions are composed of 3 discrete repeat units that match regions in $m g p B$, designated $\mathrm{B}, \mathrm{EF}$ and $\mathrm{G}$, and a composite repeat unit that matches region(s) in $m g p C$. The $m g p C$ repeat is designated JKLM $[15,17,18$, 29, 31]; see Additional file 6: Figure S4). In all fully sequenced M. genitalium genomes and across our collection where the contiguation is unambiguous, the JKLM repeat can be found in MgPar loci as a complete unit, as is found in the $m g p C$ gene locus itself, as a truncated sequence (as seen in MgPar locus1) or split (JKLM-1 and 
JKLM-2) and truncated (as found in MgPar loci 3, 4, 5 and 7). It is clear that not all MgPar regions contain all of the repeated sequences [this study; $[15,18,31]$ : The $G$ repeat is always found after the shorter JKLM or inserted between the 2 split JKLM fragments: JKLM-1 and JKLM-2 (see Additional file 6: Figure S4).

To study the dynamics of recombination in the MgPar loci and $m g p B C$ expression site genes, we postulated that we could use the disruption of phylogenetic signal for each of the multiple homologous repeat sequences within the MgPar loci and the $m g p C$ gene itself to determine the nature, extent and rapidity of recombination in these regions. To do this, we first analysed the genomes of isolates M6151, M6090 and M6312 which were collected sequentially from the same French patient over 79 days (Table 1). The whole genome data from these three isolates showed that only 7 SNPs differentiate M6312 from M6090 or M6151 (after recombination was removed). We reconstructed the phylogeny of each of the B, EF and G repeat sequences. The JKLM repeat was split so that phylogenies for JKLM-1 and JKLM-2 could be constructed independently.

Within the timeframe of the sampling of this patient we could see disruption of the phylogenies for the EF, JKLM-1 and JKLM-2 repeat sequences in different MgPar loci (Additional file 7: Figure S5). The SNP profile of the different EF repeats alongside the isolates EF repeats phylogeny (Additional file 7: Figure S5, for EF repeat, right), show in detail examples of both homologous and non-reciprocal recombination, with exchange of whole repeats and partial repeats, consistent with previous reports $[5,15,18,31]$. For example, for the $m g p B$ location, the EF repeat of isolates M6090 and M6151 are identical while in sample M6312 the repeat at the same location has sequence signatures of both the EF repeats from MgPar2 and MgPar6. This is reflected in the specific phylogeny for these 3 genomic locations (Additional file 6: Figure S4, for EF, tree on the right).

Perhaps unsurprisingly considering the complex recombination events seen in a single patient when we extended this analysis to all of the MgPar/mgpBC repeats found in all samples and locations all phylogenetic signal was lost, with no clustering by genomic location or sample or whole genome phylogenetic position, (Additional file 8: Figure S6). However, these data did imply that both intra- and inter-specific recombination occurred in isolates of the same and different clades of the tree. The only exception to this was for repeats G and JKLM-2 which in many samples showed an identical sequence at the same location (MgPar loci 3, 5 and 7). It is notable that these represent 3 of the $4 \mathrm{MgPar}$ loci where the repeat $G$ is inserted into the JKLM repeat splitting it into in the two segments $[15,17,18,32]$.

\section{Antibiotic resistance of Mycoplasma genitalium isolates}

Antibiotic resistance phenotypic profiles (measured as MICs $(\mu \mathrm{g} / \mathrm{ml})$ ) for 6 antibiotics relevant (present or past) for the treatment of M. genitalium infections and other sexually transmitted pathogens, were determined for all isolates (see Table 1). Isolates comprised seven azithromycin resistant strains $(\mathrm{MIC}>8 \mu \mathrm{g} / \mathrm{ml}$ ), two of which were multidrug resistant with accompanying moxifloxacin resistance (MIC $\geq 8 \mu \mathrm{g} / \mathrm{ml}$ ). Erythromycin and ciprofloxacin showed a much lower activity against the M. genitalium isolates than azithromycin and moxifloxacin. Doxycycline MICs would generally be considered well within the in vitro susceptible range $(\mathrm{MIC} \leq 2 \mu \mathrm{g} / \mathrm{ml})$, although one strain (M6303) had an MIC of $2 \mu \mathrm{g} / \mathrm{ml}$ (Table 1).

In an attempt to link the phenotypic resistance data with genotypic evidence of resistance, we searched for mutations known to confer antimicrobial resistance. Fig. 1 shows that for the two macrolides tested, azithromycin and erythromycin, there was concordance between MIC-based antimicrobial resistance profiles and the presence of SNPs in region $\mathrm{V}$ of $23 \mathrm{~S}$ rRNA, nucleotides positions A2058 and A2059, known to confer resistance [33]. The 23S rRNA A2059G mutation and particularly the A2058G mutation also resulted in substantially increased MICs of the new fluoroketolide solithromycin, i.e., MIC $0.25-1 \mu \mathrm{g} / \mathrm{ml}$ and MIC $2 \mu \mathrm{g} / \mathrm{ml}$, respectively. For the fluoroquinolones and correlating with ciprofloxacin and moxifloxacin resistance, the parC gene carried a characteristic fluoroquinolone resistance mutation in codon 83 that results in the amino acid substitution S83I (Fig. 1) [34]. However, although SNPs were found in $\operatorname{gyr} A B$ and parE, known to be associated with fluoroquinolone resistance in other Mycoplasmas [35], none of the SNPs were at known positions or correlated with the observed MIC-based resistance profiles.

All samples, including the reference, contained genes dhfR (MG_228) and ksgA (MG_463). The presence of these genes in other bacterial species confers resistance to trimethoprim [36] or kasgamycin [37] and chlarithromycin, [38], respectively. Although $d h f R$ is often located on mobile genetic elements in other bacterial species, both of these genes appear to be part of the core genome in M. genitalium (and other Mycoplasmas), with no evidence of lateral gene transfer.

Four isolates (M2341, M6303, M6489 and M6711) showed slightly reduced susceptibility to doxycycline (MICs of $1-2 \mu \mathrm{g} / \mathrm{ml}$; Table 1 ). We looked within the 11 ABC-family membrane transporter loci in the genome of $M$. genitalium, comprising more than 36 predicted CDSs [5, 39] to investigate the possibility of efflux related decrease susceptibility [40]. They are present in all our isolates, although there is some sequence 
variation (in this case, SNPs) that resulted in some CDSs being truncated (data not shown). Of the 4 samples showing reduced doxycycline susceptibility none possessed additional or unique $\mathrm{ABC}$-transport systems or displayed any genetic variation that correlated to phenotypic resistance, in particular, no tet $M$ gene homologs were identified.

\section{Discussion}

This study shows the first phylogenetic framework for the species Mycoplasma genitalium, encompassing isolates from three continents which were collected over three decades (1980-2010). The phylogenetic tree subdivides the species into two main clades, of which Clade $\mathrm{A}$ includes the first genome sequenced strain $\mathrm{G} 37^{\mathrm{T}}$ [5]. Linking the isolate metadata to the phylogeny, there was no obvious correlation between phylogenetic position and either country of origin or the year of isolation, with both clades containing isolates from the three continents sampled (Europe, Australia and Asia (Japan)). However, whilst this is the largest collection of $M$. genitalium sequenced more intensive sequencing from more geographically and temporally diverse sources or collections would be needed to fully answer this question.

Comparing the genome assemblies and annotation of all isolates showed that genomes varied in size $\sim 1 \mathrm{~kb}$ as an average (not excluding MgPar misassembled portions) with all isolates predicted to encode the same number of protein coding genes $(n=482)$, with no novel gene and/or other unique accessory regions with respect to the reference $\mathrm{G}_{3} 7^{\mathrm{T}}$ genome $[5,27]$. Likewise, there was no evidence of the presence of extra chromosomal elements in any of the genomes sequenced. Five of the strains sequenced, the ATCC: R32G, TW10-6G, TW105G, TW48-5G [25] and UTMB-10G [26], presented negligible differences between them and to the reference $\mathrm{G}^{2} 7^{\mathrm{T}}$ genome. It is important that the $M$. genitalium research community becomes aware of this fact. Although the clinical samples from which they were isolated were collected in 1974-5 [25] and 1986 [26], their isolation took place in 1987 in the same laboratory where $\mathrm{G} 37^{\mathrm{T}}$ was potentially used at the same time as a positive control for the growth conditions and other reference strains may have been cross-contaminated with G37 ${ }^{\mathrm{T}}$. This is certainly consistent with the sequence data, and the reason our subsequent analysis was carried out without these five sequences.

Using SNP density as a predictor of recombination it is evident that recombination appears non-uniform and as hotspots distributed across our genomes, a fact that has been shown previously [28]. Furthermore, the different sub-lineages found show different patterns of recombination. For example, Clade $B$ carries a unique recombination region stretching from gyrA to MG_009 (encompassing essential genes encoding for DNA topology, and DNA and tRNA synthesis proteins), and different regions have been subjected to different numbers of recombination events when compared across all isolates. It is clear that recombination is not restricted to MgPar loci, with other cytoadherence and lipoprotein proteins being included $[5,30]$, as well as housekeeping genes involved in DNA topology, like the region mentioned (region I) shared by clade II isolates, which includes the GyrA function.

The nine MgPar regions show the highest level of recombination, consistent with previous findings [5], with the three genomic regions showing the highest number of recombination events represented by the block composed of MgPar4, mgpABC and MgPar5, the block composed of MgPar2 and MgPar3, and the regions adjacent and containing MgPar1. Through this recombination, which we have shown as both reciprocal and non-reciprocal intramolecular events, in accordance with previous findings $[15,18,29,31]$, the MgPar regions represent a reservoir of variation that can be rapidly introduced into the expression site genes $m g p B C$, and through recombination between MgPar loci create further diversity in the MgPar loci themselves. The resulting $m g p B C$ variability translates into antigenic variation that permits $M$. genitalium to evade the host immune system in order to establish the infection and its persistence [18].

Unlike the MgPar regions, the other recombinogenic regions illustrated along the genome are not repeated and accordingly, they cannot follow the same model. Looking at all recombinogenic regions, including $m g p B C$ and the MgPar loci, based on our default mapping cut-off of $>91 \%$, all these regions share more sequence identity with orthologonal loci in M. genitalium isolate genomes than to any other Mycoplasma species. However, this may not be too surprising since it is an extremely diverse genus with orthologues in $M$. pneumoniae being the most similar sharing on average $69 \%$ nucleotide identity (our own data) or $75.9 \%$ protein similarity [4]. Therefore, these recombined sequences within the MgPar/mgpBC and elsewhere must emanate either from an unknown close relative of $M$. genitalium or from the wider diversity of the species - still not captured in the strains we currently have sequenced.

Searching for genotypic evidence of antimicrobial resistance with no evidence of the acquisition of genes associated with AMR, we looked for mutations known to confer antimicrobial resistance. These data confirmed the presence of SNPs at nucleotide positions A2058 and A2059 in region V of the $23 \mathrm{~S}$ rRNA and was in concordance with the phenotypic MIC data for the two 
macrolides tested, azithromycin and erythromycin and to other reports [33]. Similarly, for the fluoroquinolones, we confirmed a SNP in the codon 83 for the parC gene that would result in the S83I amino acid substitution, correlating with ciprofloxacin and moxifloxacin resistance [34] but no SNPs were found in gyrAB/parE specific reported positions for Mycoplasmas [35]. These data also showed a complete absence of acquired resistance genes in these isolates, which genes such as $d h f R$ and $k s g A$, conferring various resistances in other bacteria [36-38], appearing to be part of the core genome of Mycoplasma genitalium. No genetic factors have been shown to confer doxycycline resistance in M. genitalium, although for $M$. hominis it has been previously suggested that genes encoding membrane transport functions such as $\mathrm{ABC}$-family membrane transport proteins could be involved in conferring resistance to these antibiotics through efflux [40]. However, none of the four strains showing reduced susceptibility to this antibiotic displayed genetic variations correlating with it.

Although we have limited power to understand the impact of antimicrobial resistance on the population structure of $M$. genitalium in this study, when the phenotypic and genotypic resistance profiles were added to the whole genome phylogeny, it was clear that, antimicrobial resistance was not associated to a specific clade in the tree and has emerged independently on multiple occasions.

\section{Conclusion}

In this present work, we have increased the number of distinct genomes for the fastidious and slowgrowing M. genitalium from 5 to 26. All isolates were cultured and were with known provenance. This provides a much-needed snapshot of the population framework of M. genitalium collected between 1980 and 2010 and over multiple continents; it underlines the huge degree with which recombination has and is shaping the evolution of this species and not just in the MgPar loci. Avoiding the hotspots of recombination is also important when designing molecular tests for diagnosis and typing of $M$. genitalium, at a time when antibiotic resistance increases both in clinical and non-clinical settings.

\section{Methods}

M. genitalium strains, culture history and DNA extractions A total of $22 M$. genitalium isolates were obtained from 20 patients attending clinics in Europe, Asia, and Australia (Table 1) by primary inoculation into Vero cell cultures and monitoring growth by qPCR as previously described [23, 24, 41]. After a varying number of passages in the Vero cell culture system, M. genitalium was sufficiently adapted to establish growth in cell free mycoplasma medium. The isolates were single colony cloned three times to ensure purity. Three strains were isolated from the same French patient; their clinical samples were kindly provided by Dr. Bertille de Barbeyrac, Bordeaux, France. The M30 early strain was obtained in its 7th passage from The Mollicutes Collection of Cultures and Antisera, Gainesville, as a freezedried culture. This early passage of one of the two original isolates [1] is clearly distinct from the M30 strain available from the ATCC [41]. Five isolates available from the ATCC described as extra-genital (R32G, TW10-6G, TW10-5G, TW48-5G [25] and UTMB-10G [26] were obtained directly from Dr. Joseph Tully who also deposited the strains in the ATCC.

All strains were grown at $37{ }^{\circ} \mathrm{C}$ in $150 \mathrm{ml}$ of modified Friis' FB medium [24] in 800-ml Nunclon ${ }^{\text {Tx }}$ disposable polystyrene culture flasks (ThermoFisher Scientific, Waltham, MA, USA). Adherent mycoplasmas from four flasks were harvested by centrifugation in the late log phase. DNA was extracted from the cell pellets using the QIAamp ${ }^{\circ}$ DNA Mini Kit (Qiagen, Hilden, Germany) according to the manufacturer's instructions. DNA concentrations were measured with the Qubit dsDNA HS Assay Kit (ThermoFisher Scientific).

\section{Genome sequencing and de novo assemblies}

DNA libraries for all samples were prepared according to published protocols $[42,43]$ and sequenced on an Illumina MiSeq operated platform according to the manufacturer's instructions at the Wellcome Trust Sanger Institute. Total bases of raw data per sample are recorded in Additional file 1: Table S1. An average of $135.02 \mathrm{Mb}$ of sequence data was obtained per sample in $150 \mathrm{bp}$ read pairs. De novo genome assemblies were obtained from Illumina fastq files using an in-house implemented pipeline with detailed stages previously published [44] and can be downloaded from our ftp site [45]. Artemis [46] and ACT (Artemis Comparative Tool, [47]) were used as genome browsers for manual checking and comparison of genome assemblies.

\section{Mapping, single nucleotide polymorphism (SNP) calling and phylogenetic tools}

Phylogenetic analysis was performed using the isolates read data (this study) and the published [22] genomes of samples M2321, M6280, M6320 and M2288 (accession numbers [GenBank: CP003770-3], respectively). Fastq data was generated from published genomes by simulating short-read data in silico. Read mapping was performed using SMALT [48] against the sequence of M. genitalium strain $\mathrm{G}^{\mathrm{T}}{ }^{\mathrm{T}}$ (accession number [GenBank: L43967]) using a lower cut off for mapping of 91\% nucleotide identity [5, 49], ignoring exact repeats; for 
mapping parameters see Additional file 1: Table S1). Single nucleotide polymorphisms (SNPs) were identified using an in-house script as previously described [50]. Gubbins [51] was run on the whole genome alignment in order to identify regions of recombination (defined by SNP density) which were masked out so as to produce a recombination-free phylogeny $[50,52]$. The impact of recombination was also assessed using the maximum $x^{2}$ test, neighbour similarity score (NSS) and the pairwise homoplasy index (PHI) using the PhiPack package and the M. genitalium whole genome alignment [53]. Phylogenetic trees were constructed using RAxML assuming a general time reversible site model with gamma correction [54]. Support for clades in the maximum likelihood tree was assessed by running the same analysis on 100 bootstrap replicates.

M. pneumoniae strain M129 (accession number U00089), a member of the most closely related $\mathrm{Myco}$ plasma species $[27,55]$ was used to define the root position for the $M$. genitalium phylogenies by constructing a phylogeny based on core gene SNPs from a $M$. pneumoniae-M. genitalium pan-genome using Roary (v3.7.0) [56] with a minimum protein identity of $75 \%$ and genes aligned using MAFFT (v7.205) [57]. This resulted in a predicted core genome of 264 genes/ortholog groups equating to a core of 174,384 bases which is $30 \%$ of the size of $M$. genitalium genome. The corresponding alignment can be downloaded as a DOI file [58].

SEAVIEW (v.4.2), [59] was used for sequence alignments and visualization. Phylogenetic groups were defined using Bayesian Analysis of Population Structure, performed on the variant sites alignment using the BAPS individual mixture model [60].

Tanglegrams comparing tree topologies were prepared using Dendroscope (v.3.2.10) [61].

SEAVIEW, using MUSCLE, was used to align the sequences of the MgPar or $m g p B C$ loci, which were taken from each genome assembly. RAxML was used to construct bipartition trees from these alignments using parsimony, the final un-rooted phylogenetic tree was used to determine SNPs per branch [44]. Where assembled sequences of non-typical MgPar regions were not certain due to high levels of recombination, they were excluded from the analysis.

\section{Antimicrobial resistance testing}

The minimum inhibitory concentrations (MICs) of azithromycin, erythromycin, moxifloxacin, ciprofloxacin, doxycycline, and solithromycin were determined using a Vero cell culture based method as previously described $[62,63]$. SNPs associated with resistance were investigated by extracting the sequences of the relevant rRNA or housekeeping gene from each sample's assembly by using an in-silico PCR script.

\section{Additional files}

Additional file 1: Table S1. Illumina sequenced genomes of $M$. genitalium isolates and related genomes: mapping and assembly statistics and genome characteristics. (XLSX $20 \mathrm{~kb}$ )

Additional file 2: Table S2. Recombination regions identified and removed for the Mycoplasma genitalium phylogeny. (XLS 89 kb)

Additional file 3: Figure S1. Dendogram output bipartitions tangled trees representing changes on topology before (left) and after (right) running Gubbins. (PDF 124 kb)

Additional file 4: Figure S2. Bootstrapping values for the M. genitalum phylogenetic tree represented in Fig. 1. (PDF 852 kb)

Additional file 5: Figure S3. Path-O-Gen output plots. The plots are showing the root to rip distances vs. year of isolation of all Mycoplasma genitalium strains (left) and the frequency distribution after a 100 permutations (right). (PDF $6 \mathrm{~kb}$ )

Additional file 6: Figure S4. Sequence homology representation of the $m g p B$ and $m g p C$ genes repeats positions and their homologous sequences in $\mathrm{MgPar}$ regions. Homologue repeat positions in each of the different structured MgPar region are highlighted in the same colours. All based on the M. genitalium G37 genome. Dotted vertical lines represent restriction fragments and hatched boxes represents intervening sequences that are unusually A-T rich and contain stop codons [15]. (PDF $114 \mathrm{~kb})$

Additional file 7: Figure S5. RAxML Phylogenetic trees reconstructed with parsimony for the five homologous repeat sequences contained in the mgp operon and the MgPar regions for isolates of the same patient. Sequences were coloured when they were not the same in the three isolates. Numbers over the scale line represent SNPs. For the EF sequences, as an example, all SNPs with respect to the $G 37^{\top} \mathrm{EF}$ sequence at the $m g p$ operon location are plotted after reconstruction on the right. As identical samples contain identical SNPs profiles, it is easy to spot blocks of sequence replacements due to recombination. One reciprocal recombination is marked with a cross for the two locations where it happened within the same sample. Other blocks do not have reciprocal counterparts, a sign of multiple recombination steps or a unique nonreciprocal recombination event. (PDF $195 \mathrm{~kb}$ )

Additional file 8:Figure S6. RAxML Phylogenetic trees reconstructed with parsimony for the five homologous sequences contained in the mgp operon and the MgPar regions of Mycoplasma genitalium genomes. Numbers over the scale line represent SNPs. Clustering of sequencing by sample or genomic location has been emphasized by displacing rectangles within the metadata columns. (PDF $539 \mathrm{~kb}$ )

\section{Abbreviations}

\%GC: Percentage of Guanine and Cytosine content; ACT: Artemis Comparative Tool; ATCC: American Type Culture Collection; AZM: Azithromycin; CDS: Coding Sequence; CIP: Ciprofloxacin; DOX: Doxycycline; EMBL: European Molecular Biology Laboratory; ERY: Erythromycin; MIC: Minimum Inhibitory Concentration; MXF: Moxifloxacin; NAATs: Nucleic Acid Amplifications Tests; NGU: Nongonococcal Urethritis; rRNA: ribosomal RiboNucleic Acid; SNP: Single Nucleotide Polymorphism; SOL: Solithromycin

\section{Acknowledgements}

We thank Theresa Feltwell, Dr. David Harris and Team B at WTSI for their input at handling samples and sequencing, and to Team 230 also at WTSI for assistance with dataset preparations for public availability.

\section{Funding}

This study was partly supported by a grant from Aage Bangs Fond to JSJ and by the Wellcome Trust grant 098051 to authors from WTSI.

\section{Availability of data and materials}

Samples Short Read Archive accession numbers are written in Additional file 1: Table S1 and their reads data (project no. PRJEB5172) can be downloaded from EMBL Nucleotide Sequence Database, ENA, with the following direct web link: http://www.ebi.ac.uk/ena/data/view/PRJEB5172. Assemblies can be 
downloaded from our ftp site via figshare: 10.6084/m9.figshare.5285269.v1. Phylogenetic trees and their corresponding alignments were uploaded into the public repository TreeBASE (http://purl.org/phylo/treebase/phylows/ study/TB2:S21973?format=html).

\section{Authors' contributions}

NRT, MCF, JSJ and MU conceived the study and wrote the manuscript. MCF, SP and MU performed lab work. MCF, JH and SH carried out bioinformatics and analysis. All authors read and approved the final draft.

\section{Ethics approval and consent to participate}

All sequenced $M$. genitalium isolates were cultured and preserved as part of standard diagnostics and/or care, and no patient identification information was available in the study. Consequently, ethical approval was not required.

\section{Consent for publication}

Not applicable.

\section{Competing interests}

The authors declare that they have no competing interests.

\section{Publisher's Note}

Springer Nature remains neutral with regard to jurisdictional claims in published maps and institutional affiliations.

\section{Author details}

${ }^{1}$ Pathogen Genomics, The Wellcome Trust Sanger Institute, Wellcome Trust Genome Campus, Hinxton, Cambridge, UK. ${ }^{2}$ Clinical Microbiology and Public Health Laboratory, National Infection Service, Cambridge University Hospitals NHS Foundation Trust, Hills Road, Cambridge, UK. ${ }^{3}$ WHO Collaborating Centre for Gonorrhoea and other Sexually Transmitted Infections, Department of Laboratory Medicine, Microbiology, Faculty of Medicine and Health, Örebro University, Örebro, Sweden. ${ }^{4}$ Division for Infection Preparedness, Bacteria, Parasites, and Fungi, Research Unit for Reproductive Tract Microbiology, Statens Serum Institute, Copenhagen, Denmark. ${ }^{5}$ London School of Hygiene and Tropical Medicine, London, UK.

Received: 9 August 2017 Accepted: 19 December 2017

Published online: 28 December 2017

\section{References}

1. Tully JG, Taylor-Robinson D, Cole MR, Rose DLA. Newly discovered mycoplasma in the human urogenital tract. Lancet. 1981;317:1288-91.

2. Taylor-Robinson D, Jensen JS. Mycoplasma genitalium: from Chrysalis to multicolored butterfly. Clin Microbiol Rev. 2011;24:498-514.

3. Unemo M, Jensen JS. Antimicrobial-resistant sexually transmitted infections: gonorrhea and mycoplasma genitalium. Nat Rev Urol. 2017;14:139-52.

4. Himmelreich R, Hilbert H, Plagens H, Pirkl E, Li BC, Herrmann H. Complete sequence analysis of the genome of the bacterium mycoplasma pneumonia. Nucleic Acids Res. 1996;24:4420-49.

5. Fraser CM, Gocayne JD, White O, Adams MD, Clayton RA, Fleischmann RD, Bult CJ, Kerlavage AR, Sutton G, Kelley JM, Fritchman JL, Weidman JF, Small KV, Sandusky M, Fuhrmann J, Nguyen D, Utterback TR, Saudek DM, Phillips CA, Merrick JM, Tomb J-F, Dougherty BA, Bott KF, P-C H, Lucier TS, Peterson SN, Smith HO, Hutchison CA III, Venter JC. The minimal gene complement of mycoplasma genitalium. Science. 1995;270:397-403.

6. Himmelreich R, Plagens $H$, Hilbert $H$, Reiner B, Herrmann R. Comparative analysis of the genomes of the bacteria mycoplasma pneumoniae and mycoplasma genitalium. Nuc. Acids Res. 1997;25:701-12.

7. Taylor-Robinson D, Jensen JS. Mycoplasmas. In: Warrell DA, Cox TM, Firth JD, Török ME, editors. Oxford textbook of medicine: infection. Oxford University Press: Oxford; 2012. p. 571-81.

8. Lis R, Rowhani-Rahbar A, Manhart LE. Mycoplasma genitalium infection and female reproductive tract disease: a meta-analysis. Clin Infect Dis. 2015;61:418-26.

9. Jensen JS, Cusini M, Gomberg M, Moi H. European guideline on mycoplasma genitalium infections. J Eur Acad Dermatol Venereol. 2016;30:1650-6.

10. Jensen JS, Bradshaw C. Management of Mycoplasma genitalium infections can we hit a moving target? BMC Infect Dis. 2015;15:343.
11. Haggerty CL, Totten PA, Astette SG, Lee S, Hoferka SL, Kelsey SF, Ness RB. Failure of cefoxitin and doxycycline to eradicate endometrial mycoplasma genitalium and the consequence for clinical cure of pelvis inflammatory disease. Sex Trasnm Infect. 2008:84:338-42.

12. Tully JG, Taylor-Robinson D, Rose DL, Cole RM, Bove JM. Mycoplasma genitalium, a new species from the human urogenitaltract. Int J Syst Bacteriol. 1983;33:387-96.

13. Thomson N, Bentley S, Holden M, Parkhill J. Fitting the niche by genomic adaptation. Nat Revs Microb. 2003;1:92-3.

14. Zamenhof S, Eichhorn HH. Study of microbial evolution through loss of biosynthetic functions: establishment of "defective" mutants. Nature. 1967;216:456-8.

15. Ma L, Jensen JS, Mancuso M, Hamasuna R, Jia Q, McGowin CL, Martin DH. Genetic variation in the complete $\mathrm{MgPa}$ operon and its repetitive chromosomal elements in clinical strains of mycoplasma genitalium. PLoS One. 2010; 10.1371/journal.pone.0015660.

16. Wood GE, Iverson-Cabral SL, Patton DL, Cummings PK, Cosgrove-Sweeney YT, Totten PA. Persistence, immune response, and antigenic variation of mycoplasma genitalium in an experimentally infected pig-tailed macaque (Macaca Nemestrina). Infect Immun. 2013;81:2938-51.

17. Ma L, Jensen JS, Mancuso M, Hamasuna R, Jia Q, McGowin CL, Martin DH. Variability of trinucleotide tandem repeats in the $\mathrm{MgPa}$ operon and its repetitive chromosomal elements in mycoplasma genitalium. J Med Microbiol. 2012;1:191-7.

18. Iverson-Cabral SL, Astete SG, Cohen CR, Totten PA. mgpB and mgpC sequence diversity in mycoplasma genitalium is generated by segmental reciprocal recombination with repetitive chromosomal sequences. Mol Microbiol. 2007;66:55-73

19. Iverson-Cabral SL, Wood GE, Totten PA. Analysis of the mycoplasma genitalium MgpB adhesin to predict membrane topology, investigate antibody accessibility, characterize amino acid diversity, and identify functional and immunogenic epitopes. PLoS One. 2015; 10.1371/journal. pone.0138244.

20. Ma L, Taylor S, Jensen JS, Myers L, Lillis R, Martin DH. Short tandem repeat sequences in the mycoplasma genitalium genome and their use in a multilocus genotyping system. BMC Microbiol. 2008;8:130-42.

21. Hjorth SV, Björnelius E, Lidbrink P, Falk L, Dohn B, Berthelsen L, Ma L, Martin DH, Jensen JS. Sequence-based typing of mycoplasma genitalium reveals sexual transmission. J Clin Microbiol. 2006;44: 2078-83

22. McGowin CL, Ma L, Jensen JS, Mancuso MM, Hamasuna R, Adegboye D, Martin DH. Draft genome sequences of four axenic mycoplasma genitalium strains isolated from Denmark, Japan, and Australia. J Bacteriol. 2012;194:6010-1.

23. Hamasuna R, Osada $Y$, Jensen JS. Isolation of mycoplasma genitalium from first-void urine specimens by coculture with Vero cells. J Clin Microbiol. 2007:45:847-50.

24. Jensen JS, Hansen HT, Lind K. Isolation of mycoplasma genitalium strains from the male urethra. J Clin Microbiol. 1996;34:286-91.

25. Baseman JB, Dallo SF, Tully JG, Rose DL. Isolation and characterization of mycoplasma genitalium strains from the human respiratory tract. J Clin Microbiol. 1988;26:2266-9.

26. Tully JG, Rose DL, Bseman JB, Dallo SF, Lazzell AL, Davis CP. Mycoplasma pneumoniae and mycoplasma genitalium mixture in synovial fluid isolate. J Clin Microbiol. 1995:33:1851-5.

27. Pereyre $S$, Sirand-Pugnet $P$, Beven $L$, Charron $A$, Renaudin $H$, Barre $A$, Avenaud P, Jacob D, Couloux A, Barbe V, de Daruvar A, Blanchard A, Bébéar C. Life on arginine for mycoplasma hominis: clues from its minimal genome and comparison with other human urogenital mycoplasmas. PLoS Genet 2009; doi: 10.1371/journal.pgen.1000677.

28. Rocha EPC, Blanchard A. Genomic repeats, genome plasticity and the dynamics of mycoplasma evolution. Nucleic Acids Res. 2002;30: 2031-42.

29. Iverson-Cabral SL, Astete SG, Cohen CR, Rocha EPC, Totten PA Intrastrain heterogeneity of the $\mathrm{mgpB}$ gene in mycoplasma genitalium is extensive in vitro and in vivo and suggests that variation is generated via recombination with repetitive chromosomal sequences. Infect Immun. 2006;74:3715-26.

30. Waldo RH, Popham PL, Romero-Arroyo CE, Mothershed EA, Lee KK, Krause DC. Transcriptional analysis of the hmw gene cluster of mycoplasma pneumonia. J Bacteriol. 1999;181:4978-85. 
31. Ma L, Jensen JS, Myers L, Burnett J, Welch M, et al. Mycoplasma genitalium: an efficient strategy to generate genetic variation from a minimal genome. Mol Microbiol. 2007;66:220-36.

32. Hu PC, Schaper U, Collier AM, Clyde WA Jr, Horikawa M, Huang Y-S, Bariles MF. A mycoplasma genitalium protein resembling the mycoplasma pneumoniae attachment protein. Infect Immun. 1987:55:1126-31.

33. Touati A, Peuchant O, Jensen JS, Bébéar C, Pereyre S. Direct detection of macrolide resistance in mycoplasma genitalium isolates from clinical specimens from France by use of real-time PCR and melting curve analysis. J Clin Microbiol. 2014;52:1549-55.

34. Shimada Y, Deguchi T, Nakane K, Masue T, Yasuda M, Yokoi S, Ito S-I, Nakano M, Ito S, Ishiko H. Emergence of clinical strains of mycoplasma genitalium harbouring alterations in ParC associated with fluoroquinolone resistance. Int J Antimicrob Agents. 2010;36:255-8.

35. Bébéar CM, Renaudin J, Charron A, Renaudin H, de Barbeyrac B, Schaeverbeke T, Bébéar C. Mutations in the gyrA, parC, and parE genes associated with fluoroquinolone resistance in clinical isolates of mycoplasma hominis. Antimicrob Agents Chemother. 1999;43:954-6.

36. Brisson N, Hohn T. Nucleotide sequence of the dihydrofolate-reductase gene borne by the plasmid R67 and conferring methotrexate resistance. Gene. 1984;28:271-5.

37. Sparling PF, Ikeya Y, Elliot D. Two genetic loci for resistance to Kasugamycin in Escherichia Coli. J Bacteriol. 1973;113:704-10.

38. Phunpruch $S$, Warit $S$, Suksamran R, Billamas $P$, Jaitrong $S$, Palittapongarnpimb P, Prammananan T. A role for $16 \mathrm{~S}$ rRNA dimethyltransferase ( $\mathrm{ksgA})$ in intrinsic clarithromycin resistance in mycobacterium tuberculosis. Int J Antimicrob Agents. 2013;41:548-51.

39. Paulsen IT, Sliwinski MK, Saier MH Jr. Microbial genome analyses: global comparisons of transport capabilities based on phylogenies, bioenergetics and substrate specificities. J Mol Biol. 1998;3:573-92.

40. Raherison S, Gonzalez P, Charron H, Bébéar C, Bébéar CM. Evidence of active efflux in resistance to ciprofloxacin and to Ethidium bromide by mycoplasma hominis. Antimicrob Agents Chemother. 2002;46:672-9.

41. Jensen JS, Bjornelius E, Dohn B, Lidbrink P. Use of TaqMan 59 nuclease real-time PCR for quantitative detection of mycoplasma genitalium DNA in males with and without urethritis who were attendees at a sexually transmitted disease clinic. J Clin Microbiol. 2004;42:683-92.

42. Quail MA, Kozarewa I, Smith F, Scally A, Stephens PJ, Durbin R, Swerdlow H, Turner DJ. A large genome center's improvements to the Illumina sequencing system. Nat Methods. 2008;12:1005-10.

43. Quail MA, Otto TD, GU Y, Harris SR, Skelly TF, McQuillan JA, Swerdlow HP, Oyola SO. Optimal enzymes for amplifying sequencing libraries. Nat Methods. 2011;1:10-1.

44. Bronowski C, Fookes MC, Gilderthorp R, Harris SR, Phiri A, Hall N, Gordon MA, Wain J, Hart CA, Thomson NR, Winstanley C. Genomic characterisation of invasive non-typhoidal Salmonella enterica Subspecies enterica Serovar Bovismorbificans isolates from Malawi. PLos Neglect Trop Dis. 2013; 10.1371/journal.pntd.0002557.

45. Page A: Mycoplasma genitalium annotated assemblies. figshare. 10.6084/ m9.figshare.5285269.v1 (2017). Accessed 8 Aug 2017.

46. Carver T, Harris SR, Berriman M, Parkhill J, McQuillan JA. Artemis: an integrayed platform for visualization and analysis of high-throughput sequence-based experimental data. Bioinformatics. 2012;28:464-9.

47. Carver TJ, Rutherford KM, Berriman M, Rajandream MA, Barrell BG, Parkhill JACT. The Artemis Comarison tool. Bioinformatics. 2005; 21:3422-3.

48. Ponstingl H. SMALT version 0.7.6. https://sourceforge.net/projects/smalt/ (2014). Accessed 4 Sep 2014.

49. Murray GG, Wang F, Harrison EM, Paterson GK, Mather AE, Harris SR, Holmes MA, Rambaut A, Welch JJ. The effect of genetic structure on molecular dating and tests for temporal signal. Methods Ecol Evol. 2015; 10.1111/2041-210X.12466.

50. Harris SR, Feil EJ, Holden MT, Quail MA, Nickerson EK, Chantratita N, Gardete S, Tavares A, Day N, Lindsay JA, Edgeworth JD, de Lencastre H, Parkhill J, Peacock SJ, Bentley SD. Evolution of MRSA during hospital transmission and intercontinental spread. Science. 2010; 327:469-74

51. Croucher NJ, Page AJ, Connor TR, Delaney AJ, Keane JA, Bentley SD, Parkhill J, Harris SR. Rapid phylogenetics analysis of large samples of recombinant bacterial whole genome sequences using Gubbins. Nucleic Acids Res. 2014; 10.1093/nar/gku1196.
52. Li H, Handsaker B, Wysoker A, Fennell T, Ruan J, Homer N, Marth G, Abecasis G, Durbin R, 1000 genome project data processing subgroup. The sequence alignment/map (SAM) format and SAMtools. Bioinformatics. 2009:25:2078-9.

53. Bruen T, Philippe $H$, Bryant D. A Simple and robust statistical test for detecting the presence of recombination. Genetics. 2006;172:2665-81.

54. Stamatakis A. RAxML-VI-HPC: maximum likelihood-based phylogenetic analyses with thousands of taxa and mixed models. Bioinformatics. 2006;22:2688-90.

55. Lin Y, Zhang RR. Putative essential and core-essential genes in mycoplasma genomes. Sci Rep. 2011;1:53.

56. Page AJ, Cummins CA, Hunt M, Wong VK, Reuter S, Holden MTG, Fookes M, Falush D, Keane JA, Parkhill J. Roary: rapid large-scale prokaryote pan genome analysis. Bioinformatics. 2015;31:3691-3.

57. Kazutaka K, Hiroyuki T. Recent developments in the MAFFT multiple sequence alignment program. Brief Bioinform. 2008;9:286-98.

58. Page A: Core gene alignment of Mycoplasma genitalium isolates with Mycoplasma pneumoniae outgroup. figshare. 10.6084/m9.figshare. 4524536.v3 (2017) Accessed 8 Aug 2017.

59. Galtier N, Gouy M, Gautier C. SEAVIEW and PHYLO_WIN: two graphic tools for sequence alignment and molecular phylogeny. Comput Biosci. 1996:12:543-8.

60. Corander J, Marttinen P, Siren J, Tang J. Enhanced Bayesian modelling in BAPS software for learning genetic structures of populations. BMC Bioinformatics. 2008;9:539-52.

61. Huson DH, Scornavacca C. Dendroscope 3-an interactive viewer for rooted phylogenetic trees and networks. System Biol. 2012;6:1061-7.

62. Hamasuna R, Osada Y, Jensen JS. Antibiotic susceptibility testing of mycoplasma genitalium by TaqMan 5 ' nuclease real-time PCR. Antimicrob Agents Chemother. 2005;49:4993-8.

63. Jensen JS, Fernandes $P$, Unemo M. In Vitro activity of the new fluoroketolide solithromycin (CEM-101) against macrolide-resistant and - susceptible mycoplasma genitalium strains. Antimicrob Agents Chemother. 2014;58:3151-6.

\section{Submit your next manuscript to BioMed Central and we will help you at every step:}

- We accept pre-submission inquiries

- Our selector tool helps you to find the most relevant journal

- We provide round the clock customer support

- Convenient online submission

- Thorough peer review

- Inclusion in PubMed and all major indexing services

- Maximum visibility for your research

Submit your manuscript at www.biomedcentral.com/submit

) Biomed Central 